\title{
Sleep-wake patterns reported by parents in hyperactive children diagnosed according to ICD-10, as compared to paired controls
}

Running head: Sleep patterns reported by parents in hyperactive children compared to controls

Authors: Gomes, Ana Allen* (1; 2); Parchão, Carla (1; 3); Almeida, Anabela (1); Clemente, Vanda (4); Azevedo, Maria Helena (5)

(1) Affiliation: University of Aveiro. Department of Education. Address: Campus Universitario de Santiago. 3810-193 Aveiro, Portugal.

(2) Affiliation: IBILI (FM-UC). Address: Azinhaga de Santa Comba - Celas. 3000-548 Coimbra, Portugal.

(3) Affiliation: Department of Children and Adolescents Mental Health, Magalhães Lemos Hospital, Oporto Hospital Centre. Address: Rua Professor Álvaro Rodrigues. 4149-003 Porto, Portugal.

(4) Affiliation: Sleep Medicine Centre, CHUC (Centro Hospitalar e Universitário de Coimbra). Address: Address: Quinta dos Vales. São Martinho do Bispo. 3046-853 Coimbra, Portugal.

(5) Affiliation: Faculty of Medicine, University of Coimbra. Address: Rua Larga. 3004504 Coimbra, Portugal.

*Corresponding author: Ana Allen Gomes. E-mail address: ana.allen@ua.pt. Phone: $(+351) 234 \quad 370$ 353.Fax: (+351) $234 \quad 370$ 640. (Other authors' emails: cparchao@hotmail.com; psic.anabelaalmeida@gmail.com; mazevedo@,fmed.uc.pt; vandaclem@gmail.com). 


\begin{abstract}
This study aimed primarily to compare the parent-reported sleep of children with ICD-10 hyperkinetic disorder (HKD) versus community children. Thirty children aged 5 to 13 years ( $83.3 \%$ boys) diagnosed with HKD by their child and adolescent psychiatrists took part in this study, plus 30 community children, matched for sex, age, and school year. Compared to the controls, the HKD children showed significantly later bedtimes, stronger bedtime resistance, longer sleep latency, shorter sleep; more frequent behaviors and symptoms concerning falling asleep into parents bed, needing something special to initiate sleep, nightmares, sleep talking, sleep bruxism, fear from darkness, bedwetting, and, most notably, loud snoring (26.7\%); they also tended to show higher daytime somnolence. ADHD/HKD children may thus have more sleep-related problems than typically developing children. Alternatively, our results may reflect misdiagnoses; thus, special attention should be directed to comorbidity and differential diagnosis issues between sleep disturbances and ADHD/HKD.
\end{abstract}

Keywords: hyperactivity/inattention; sleep; hyperkinetic disorder; ADHD; children

\title{
Introduction
}

Attention deficit/hyperactivity disorder (ADHD) and hyperkinetic disorder (HKD) are terms used, respectively, by the Diagnostic and Statistical Manual, $4^{\text {th }}$ edition (DSMIV) [1] and by the International Classification of Diseases, $10^{\text {th }}$ edition (ICD-10) [2], to describe one of the most common childhood mental disorders -- childhood hyperactivity. This disorder is characterized by three main groups of symptomatology: overactivity, impulsivity, and inattention. Despite various similarities and overlaps between the two systems of classifying mental disorders, it is usually accepted that the HKD defined by ICD-10 is a more severe form of the ADHD syndrome described by the DSM-IV (see, e.g., $[3,4]$ ), most probably corresponding to the ADHD-combined 
type (see, e.g., [5]). Therefore, unsurprisingly, due to the more restrictive and more numerous criteria, HKD is usually found to have a lower prevalence than ADHD [4, 6, 7].

Some clinicians and researchers sense that sleep-related problems and complaints are relatively common in children with hyperkinetic/attention deficit hyperactivity disorder (HKD/ADHD). Thus, there has been a growing number of studies about sleep and either diagnosed ADHD or ADHD symptoms (e.g., [8] and the reviews or meta-analysis from $[9,10,11,12])$, reflecting a renewed interest in this topic. Thus it is now well documented that sleep difficulties and HKD/ADHD symptoms/diagnosis are often associated. Research on this topic is relevant, not only from a theoretical perspective, but also because of its potential impact on daily clinical practice (see [9] review).

Studies using subjective methods (see, e.g., $[9,10,12]$ ) have commonly reported that, in comparison with the control groups, children with ADHD diagnosis/symptoms usually show stronger bedtime resistance, have later bedtimes, have more difficulties with sleep onset (e.g., longer sleep latency; needing special activities/objects to initiate sleep; falling asleep in the parents bed) or symptoms of insomnia; shorter sleep length (depending on the study); have more sleep-related breathing difficulties or disorders; have more frequent symptoms of sleep talking, being afraid of the dark, nightmares, snoring, etc; and have a higher level of daytime somnolence. (It is noted that during the daytime, ADHD children may exhibit deficits in alertness and somnolence, and it has even been proposed that excessive motor activity is a way of trying to stay alert and awake.) Children with ADHD had poorer quality sleep according to objective records, too. These included the latency of onset of sleep, the number of shifts in the stages of sleep, the apnea-hypopnea index, sleep efficiency, the average time to fall asleep (as measured by the Multiple Sleep Latency Test, MSLT), as well as excessive somnolence 
during the day (also as measured by the MSLT; cf. $[9,10])$. Studies have also regularly documented ADHD/ADHD-symptoms to be associated with restless legs syndrome (RLS) and RLS symptoms, periodic limb movement disorder (PLMD) and PLMD symptoms, and excessive nocturnal motor activity, such as in the arms or legs (see, e.g., $[9,13])$. Although objective sleep studies do not always support parental reports (e.g., $[13,14,15])$, according to Konafal et al. [9], this is not surprising for the following reasons. First, objective studies typically monitor sleep for only a single night. This will capture sleep-related difficulties that occur on a nightly basis, but may well miss those that occasionally or frequently skip a night. This is important because, typically, ADHD children show a high night-to-night variability in their sleep patterns. Second, objective measures are intended to identify physiopathology, not sleep-related behaviors, such as bedtime resistance, which is better captured by subjective measures. A very few studies have considered the differences in sleep-wake patterns between the different ADHD subtypes, as defined by the DSM-IV [16]. The inattentive (I) type was consistently associated with daytime sleepiness $[17,18,19,20]$, or with hypersomnia and inadvertent napping [21] (inadvertent napping is also associated with the combined subtype); whereas in some studies, those with predominantly hyperactive-impulsive (HI) symptoms showed higher bedtime resistance [16, 18], a higher risk of snoring [17], and higher rates of specific sleep disorders [16], including daytime sleepiness and a tendency for sleep-disordered breathing. According to a large scale study [21], compared to the controls, those with the combined (C) ADHD subtype had significantly higher rates of circadian rhythm problems, sleep-talking, and nightmares (also present in the HI subtype).

Another topic of interest when addressing sleep in children with ADHD concerns the medications used to manage the disorder. Associations found between troubled sleep 
and ADHD medications, especially psychostimulants, have been inconsistent between studies, and are still poorly understood [22, 23, 24, 25]. Whereas some studies report more sleep troubles in medicated than in unmedicated ADHD children, others find few or no differences [22]; and while some studies suggest that stimulant medication has adverse effects on sleep (at least in the short term), others consider that, when treated with stimulant medication, sleep and daytime sleepiness improve or normalize in ADHD children. According to Cortese et al. [24], on behalf of the European ADHD Guidelines Group, no current meta-analyses are available on the effects of long-acting psychostimulants, and it is difficult to pool the results of the available studies due to the heterogeneity of the substances, formulations, dosages, and timetables. Thus, the existing guidelines for the management of sleep disorders associated with ADHD are still not properly evidence based [23]. In summary, it seems that although medications may have some unwanted effects on sleep - especially in the short term - this deserves further investigation (in particular, for methylphenidate), and it is probable that the poor sleep that is reported for medicated ADHD children is principally caused by the severity of the ADHD itself (e.g., $[23,24])$, which leads to the prescription of medication, and comorbidity.

For the moment, it is still uncertain whether sleep problems are intrinsic to, or comorbid conditions with, HKD/ADHD, or even if they generate HKD/ADHD-like symptoms, thus leading to misdiagnoses (cf. $[12,26])$. In medicated children, sleeprelated complaints may also arise as side effects. That is, although one may assume a bidirectional relationship between sleep disturbances/disorders and ADHD/HKD [27], the nature of the association remains unclear [12]. In all cases, since sleep-related problems may exacerbate the severity of ADHD or even produce ADHD-like symptoms, it is important to assess the quality of sleep in children diagnosed with or 
suspected of having $\mathrm{HKD} / \mathrm{ADHD}$. It is likely that, in many cases, appropriate management of sleep disturbances would contribute to the quality of life of these children and their caregivers.

Despite numerous studies reporting associations between sleep and ADHD symptoms, we believe it is necessary and pertinent to continue to investigate this topic. We note that the research findings are not consistent (for instance, augmented sleep length in ADHD children is found in some studies, e.g., [28]). Furthermore, most published studies on this topic address the symptoms of ADHD, and only a few of the available articles are based on children with a clinical diagnosis of ADHD. More importantly, professionals may not always consider the possibility of difficulties with sleep when assessing children referred to them for evaluation due to $\mathrm{ADHD} / \mathrm{HKD}$-like symptoms or when treating children with a confirmed diagnosis of ADHD or HKD [12, 29, 30]. Finally, very few sleep studies to date (and perhaps none in recent years) have considered clinical samples of hyperactive children using the ICD-10 diagnostic criteria, i.e., clinical samples identified as having a diagnosis of HKD.

The primary aim of the present study is to compare sleep, as reported by parents, in children diagnosed with $\mathrm{HKD}$ according to ICD-10 versus in community children matched for age, sex, and school year. A secondary aim is to compare sleep-wake patterns in HKD children medicated with methylphenidate versus those not taking any medication.

\section{Methods}

\section{Participants}

The clinical sample was composed of 30 children, $25(83.3 \%)$ boys and $5(17.6 \%)$ girls, with ages ranging from 5 to 13 years $(M=7.5$ years old, $\mathrm{SD}=2.22)$, mostly from $1^{\text {st }}$ to 
$6^{\text {th }}$ grades (except three in preschool and one in $7^{\text {th }}$ grade $)(\mathrm{M}=2.20, \mathrm{SD}=2.02)$, all $d e$ novo cases with a diagnosis of HKD according to the ICD-10 criteria, as assessed by their child and adolescent psychiatrists (all working at the Department of Child Mental Health at a Central Hospital), and with IQs higher than 80. From these children, 13 $(43.3 \%)$ were medicated by their respective child and adolescent psychiatrist with methylphenidate.

During the same academic year, a comparable sample of community controls was also selected, comprising 30 children, each one matched as far as possible for sex, age, and school year, to a correspondent child in the clinical sample. A perfect match was attained for 24 pairs of children; and 6 pairs were partially matched, that is, they had the same sex and either the same age or the same school year (the largest misalignment was merely 1 year in one variable, and there were no differences within each pair as to having passed/failed the previous school year). Thus, we were able to compose a comparable community sample comprising 5 girls and 25 boys, with ages ranging from 6 to 12 years $(M=7.5$ years old; $S D=2.11)$, from $1^{\text {st }}$ to $6^{\text {th }}$ grade in school $(M=2.27$, $\mathrm{SD}=1.70)$. The two groups $-\mathrm{HKD}$ children and community children - may be considered equivalent with regard to sex, age, and school year, as they showed exactly the same sex distribution, and there were neither relevant nor statistically significant mean differences in terms of their age $(t=0.000$; d.f. $=58, \mathrm{p}=1.0)$ or school year $(\mathrm{t}=$ 0.138, d.f. $=58, \mathrm{p}=.891)$

\section{Instruments}

The Child Sleep-Waking Questionnaire [31] was used to measure the children's patterns of sleeping and waking. It is a parental questionnaire from Portugal, and it is designed to assess the child's sleeping and waking behaviours; it was previously validated in earlier studies (details and the English language version may be found in [32] and [33]). 
Questions include sleep/wake times, total sleep time, and nighttime sleep-related behaviors, most of which are rated on a four-point scale: "never" (coded as 1) to "always" (coded as 4). In addition, parents were asked whether the child had any sleep problems, whether they have sought professional help for a sleep problem in the child, about the use of sleep medications, and to give information on their children's medical/neurological conditions. We added a question about naps, rated according to the same four-point frequency scale described above.

The standardized Portuguese versions of the Wechsler Intelligence Scales were used to measure the intelligence of the children diagnosed with HKD; in particular, depending on the age of each child, we used the Portuguese Wechsler Intelligence Scale for Children, $3^{\text {rd }}$ edition (WISC-III) [34] or the Portuguese Wechsler Preschool and Primary Scale of Intelligence-Revised (WPPSI-R) [35].

\section{Procedure}

Permission to conduct the study was requested from the ethics commission and the child mental health department at the hospital where the sample was collected.

All children who had, in their clinical file, a diagnosis of HKD (code F90 in the ICD-10 [2]) from a child and adolescent psychiatrist, were recruited for the clinical sample. The ICD-10 [2] is the system used at this department for classifying mental disorders, and the diagnoses were based on anamnesis, supplemented, when necessary, by video recordings of the child's behavior in their natural contexts (e.g., school and home). The team of child and adolescent psychiatrists was independent from the research team.

All of the children in the clinical sample were de novo cases at that hospital. About 2 to 3 months after each child was diagnosed with HKD, as the parents arrived with their child for a routine psychological assessment session, one of the members of the research team invited them to participate in the sleep study. After giving informed consent, the 
parents anonymously filled out the sleep questionnaire. In order to assure confidentiality, the completed questionnaires were archived separately from the hospital clinical file, and they were given an independent code number. As part of the routine psychological assessment session, all children were assessed for intelligence, and those with intelligence scores above the normal average range were excluded from our study. From a total of $41 \mathrm{HKD}$ children who attended the routine psychological assessment session, 2 parents refused to participate, and a total of 9 cases were excluded from the sample for various reasons: incomplete or incorrect completion of the questionnaire ( 2 cases), questionnaires were completed by a grandmother/grandfather not living with the child ( 3 cases), or because the child's IQ score was below 80 (4 cases).

The control group was randomly selected from a larger school sample $(\mathrm{n} \sim 600)$. After parental informed consent was obtained, data was collected during the same period (Jan-May 2011) for a parallel research study on sleep and ADHD symptoms that was approved by the Portuguese Ministry of Education [36]. First, for each child of the clinical sample, we determined a corresponding subset of children from the community, with the same sex, age, and school year (or contiguous age or school year, if necessary). Then, from this subset, one or more cases were randomly selected.

The data were statistically analyzed using SPSS for Windows. Given the nature of the variables (ordinal, in most cases), the distributions which did not always fit the normal curve, and because of the relatively small sample size, we chose to use medians as a measure of the central tendency of the sleep-wake patterns reported by the parents. The means were also determined, as they are more informative. Inferential analyses were done using nonparametric statistics. Mann-Whitney tests were used to compare the group median and mean. Fisher exact tests were used to compare proportions between groups. 


\section{Results}

HKC children vs Matched Controls. According to the median and mean values compared using the Mann-Whitney test (see first half of Table 1), compared to the controls, HKD children had significantly later bedtimes both on school nights and on weekend nights (median differences of 15 and 30 min later, respectively), comparable rise times, and significantly shorter length of sleep ( 1 hour less on school nights and 30 min less on weekend nights). HKD children also showed significantly less willingness to go to bed and more bedtime refusal. They fell asleep more often in their parents' bed, less frequently in their own bed, more often required the presence of their parents in their room, took a significantly longer time to fall asleep, had a higher need for comforting activities or objects, and a higher tendency for needing the lights to be on. In addition, the frequency of loud snoring, bed wetting, nightmares, sleep talking, teeth grinding, and being afraid of the dark, were all significantly higher in HKD children (who also tended to show a higher frequency for sleepwalking and night terrors). Although the frequency of naps was similar in both groups, children with HKD showed a trend towards higher daytime somnolence, and, on average, manifested significantly higher fatigue and irritability than did the matched controls.

After grouping answers and setting cut-off points to define frequent and/or probable sleep difficulties, we were able to observe consistent patterns (second half of Table 1). Significantly higher proportions of children in the clinical sample had frequent symptoms that were suggestive of sleep difficulties, such as bedtime reluctance and refusal, needing special activities or objects to fall asleep, having difficulties initiating sleep on their own in their own bed, falling asleep in their parents' bed, taking more than 30 min to fall asleep, loud snoring, bed wetting, nightmares, sleep talking symptoms 
and being afraid of the dark (there was also a nonsignificant trend towards more night terrors and difficulties in the autonomous resumption of sleep). Perceived sleep problems, as reported by parents, were also significantly more common in children with HKD than in the controls. However, compared to the parents of the controls, only slightly more parents of children with HKD had sought professional help for their child's sleeping problems. The difference was not statistically significant; similar results were found for the frequent use of medication to promote sleep.

Medicated vs unmedicated HKD children. By comparing the mean and median values (first half of Table 2, irrelevant results not shown), we see that HKD children taking methylphenidate, compared to unmedicated ones, showed a very clear trend, which was close to the significance level, towards later bedtimes (30 min later on week nights and 45 min on weekend nights), significantly lower willingness to go to bed, and trends near the significance level towards higher bedtime refusal and more often needing lights on in order to fall asleep. Interestingly, medicated children tended to be able more often to return to sleep by themselves than did unmedicated HKD children, even though parents reported them as having a similar number of awakenings during the night. There were trends suggesting more frequent nightmares and being afraid of the dark in medicated children, which was consistent with the previously mentioned higher tendency for needing lights on in order to fall asleep. Albeit not reaching statistical significance, both the average daytime fatigue and the median somnolence seemed higher in medicated HKD children.

After grouping answers and adopting cut-off points to define frequent and/or probable sleep difficulties, the percentages of affected medicated and unmedicated children were compared (Fischer exact test, cf. second half of Table 2). Only two statistically significant differences were found, in addition to five clear trends that were not 
significant. All of these were in line with the previous median/mean comparisons, that is, in the medicated subgroup there were more children resisting bedtime, needing lights on to fall asleep (nonsignificant trends), and exhibiting frequent nightmares (significant difference), whereas difficulties in the autonomous resumption of sleep were more common among unmedicated children. For the remaining night behaviors, movements, and fears, the percentages were marginally higher in medicated children. There was a significantly higher percentage of medicated children described as having a sleep problem (more than half), and an evidently higher proportion (albeit not significant) whose parents had ever sought help for the child's sleep problem, but no visible or statistically significant difference was found regarding the use of medication for sleep.

\section{Discussion}

In the present study, we compared a sample of children who had been diagnosed with HKD, according to ICD-10 criteria, by their child and adolescent psychiatrists, with a community sample that was randomly selected from a larger school sample, each one matched for sex, and for similar age and school year.

In agreement with what was seen in the existing literature using parental sleep questionnaires (e.g., [37, 38], and $[9,10,12]$ reviews/meta-analysis), in comparison to the community sample, our HKD children showed stronger bedtime resistance, later bedtimes, and more sleep-onset difficulties; slept for less time (mixed findings for this have been reported in the literature); showed a tendency to higher daytime somnolence and fatigue, had more frequent symptoms of parasomnias and other sleep complaints, such as sleep talking, nocturnal enuresis, sleep bruxism, being afraid of the dark, and, most notably, nightmares, and loud snoring as defined by Ferreira et al. [32]).

Bedtime resistance and late bedtimes may be due to behavioral problems or even 
comorbid behavioral disorders, which are common in children with HKD/ADHD. That is, sleep-limit difficulties may be an extension of the children's problems during the day, rather than a separate sleep-onset problem [33]. However, this is not necessarily the case. Two comprehensive reviews $[12,39]$ concluded that in ADHD, there may be a circadian sleep disorder associated with a phase delay, which would explain later bedtimes and resistance. In fact, interestingly, in the study of Hvolby et al. [37], comorbid oppositional defiant disorder in addition to ADHD did not show an added effect on problematic behaviour around bedtime. Weiss and Salpekar [39] suggested that bedtime reluctance and longer sleep latency may not be due to defiant behavior, but instead may simply reflect a normal reaction of a child forced to go to bed without having any subjective experience of being sleepy. According to them, many ADHD children experience a hyperalert state while in bed, and complain that they "cannot turn their thoughts off". They highlight the importance of obtaining the point of view of the child in order to determine the appropriate etiology of the sleep behavior and, therefore, the most suitable intervention strategies.

Nightmares are typical of the REM stage of sleep. The higher frequency of nightmares reported in our HKD children (which is in line with other studies, e.g., [37, 38, 40]), may be due to an increase in REM sleep, which has been found in some polysomnograpy (PSG) studies on ADHD children, particularly for those with the ADHD combined type [28]. One of the most salient results was the high frequency of loud snoring, found in at least a quarter of HKD children $(26.7 \%)$. This percentage is much higher than expected, based on either the estimated prevalence of primary snoring (i.e., without sleep apnea) in children, which is $10 \%$ to $12 \%$ according to the International Classification of Sleep Disorders, $2^{\text {nd }}$ edition (ICSD-2 [41]), or the estimated prevalence in Portugal (which, using the same criteria, is $8.6 \%$ in community 
children [32] and $12.5 \%$ in children with autistic spectrum disorders within the same age group, cf. [42]). Sleep-related breathing difficulties (e.g., [17] and reviews from [9, $10,12])$, and unusually higher frequencies of loud and/or frequent snoring in association with ADHD diagnosis/symptoms (e.g., $[30,38]$ ) have been repeatedly found in sleep studies based on parental questionnaires. Although snoring may occur without sleep apnea (primary snoring), it is a common symptom of a sleep breathing disorder, particularly of obstructive sleep apnea (OSA), whose pediatric form is present in $2 \%$ of otherwise healthy children (cf. ICSD-2 [41]). According to the ICSD-2, snoring (usually loudly) and/or difficulty breathing during sleep are part of the history of most children with OSA, and they constitute the first diagnostic criteria for pediatric OSA. Therefore, the percentage of frequent loud snoring found in our sample of children diagnosed with HKD suggests an intriguing higher prevalence of a probable sleep-related breathing disorder. Moreover, compared to the controls, our HKD children tended to show more daytime somnolence, bedwetting, and night terrors symptoms. This is in agreement with some of the features commonly present in (or precipitated by) pediatric OSA, according to the ICSD-2 [41], e.g., secondary enuresis, excessive daytime sleepiness, hyperactivity, and night terrors.

It has been repeatedly reported that a sleep-related breathing disorder, particularly obstructive sleep apnea (OSA), may mimic ADHD symptoms (see, e.g., [12]). Although OSA children are regularly studied for ADHD symptoms (see, e.g., [43, 44]), apparently much less research has examined the prevalence of sleep disordered breathing (SDB) in ADHD-diagnosed samples Thus, it is less clear whether children diagnosed with ADHD have a higher probability of having SDB. Despite the limited number of studies, the results seem consistent: a meta-analysis [10] focused on studies adopting rigorous DSM criteria found that, at least when moderate objective apnea 
symptoms are taken into account, the research findings support the notion that SDB may be more frequent in ADHD diagnosed children than in controls, and therefore deserves clinical attention. In three studies identified in a comprehensive review [12], ADHD in children appears to be associated with more habitual snoring and increased apnea-hypopnea index and respiratory disturbance index values.

Apparently, none of the children from our clinical sample who were identified by the parental questionnaire to have frequent loud snoring have been further examined for a possible sleep-related breathing disorder by the health professional responsible for their diagnose and therapy - at least, as of the date of the psychological examination for the present study, during which we collected data on sleeping and waking patterns. Our results suggest that, even though sleep complaints would often be reported if parents were asked about it, health professionals commonly refer children for assessment, diagnosis, and treatment of ADHD/HKD without first assessing their sleep patterns or ruling out the possible existence of sleep problems, either as comorbid conditions to $\mathrm{ADHD} / \mathrm{HKD}$ or as primary conditions underlying the ADHD/HKD symptoms.

The fact that in our sample about a quarter of HKD children were described by their parents as having loud snoring, raises the possibility of a faux diagnosis, a regular concern that may be found in sleep literature. Therefore, medical doctors, psychologists, and other professionals involved with the diagnosis and treatment of ADHD/HKD should be aware of the associations between ADHD/HKD symptoms and sleep, in particular sleep-related breathing problems, and the resulting implications for clinical practice. If parents report such symptoms, children should be further evaluated specifically for sleep-related breathing disorders. Since a sleep-related breathing disorder may cause ADHD/HKD-like symptoms, or at least contribute to the severity of symptoms in a child with $\mathrm{ADHD} / \mathrm{HKD}$, it may well deserve direct clinical attention and 
treatment. In conclusion, the possibility of comorbidity, or differential diagnosis, should always be considered when loud snoring is reported by parents of a child referred for a mental health assessment due to $\mathrm{ADHD} / \mathrm{HKD}$ symptoms. As the main causes of childhood sleep apnea are enlarged adenoids and tonsils, their surgical removal has been the most frequently designated treatment. A recent large, multicenter, randomized controlled trial in children with OSA (the Childhood Adenotonsillectomy Trial, CHAT), found significant improvements at 7 months of follow-up for the early adenotonsillectomy treatment condition (versus watchful waiting with supportive care) regarding sleep polysomnographic measures, teacher reports of behavior, and caregiverreported measures of executive function, behavior, and sleep apnea symptoms [45]. Further longitudinal studies are necessary in order to examine, in children diagnosed both with $\mathrm{ADHD} / \mathrm{HKD}$ and with a sleep-related breathing disorder, the course of ADHD/HKD symptoms before and after appropriate treatment of the sleep-related breathing condition.

Although the frequency of naps was similar in both groups, during daytime hours, the HKD children were described as significantly more fatigued and irritable than the matched controls. They also showed a nonsignificant trend towards higher somnolence which is in accordance with other studies of ADHD, and has been typically associated with the inattentive subtype $[17,18,19,20]$. We note that the higher somnolence may also be a symptom of an underlying sleep-related breathing disorder (cf. [41]).

Compared to unmedicated HKD children, HKD children taking methylphenidate tended to present later bedtimes and more bedtime resistance and nightmares, but appeared to more easily return autonomously to sleep; this is consistent with the possible effects on sleep of psychostimulants in ADHD children, as summarized in a recent review [9]. Given that the statistically significant differences may be scarce due to the small sample 
size, we looked for nonsignificant trends in order to minimize the probability of type-II errors. No other sleep-related differences were found between the two HKD subgroups, which supports the suggestion that sleep disorders are not exclusively associated with stimulant medication (cf. [12]). It has been proposed that a probable explanation behind the higher number of sleep-related troubles in medicated children is primarily due to the severity of their ADHD or to a comorbidity, rather than due to the medication per se [23]. It is possible that, among our HKD children, those who had been prescribed methylphenidate had more severe HKD symptomatology or comorbid conditions (which lead to the prescription of methylphenidate). More research is needed, in particular, longitudinal studies that monitor sleep at the baseline (before medication) and at several points in time after starting the treatment (with medication). Since ADHD children may exhibit sleep disturbances before being medicated, it has been recommended (by the European ADHD Guidelines Group [24]) that clinicians should carefully assess sleep at baseline, in order to avoid attributing disturbances to the prescribed drug when, in fact, they may be due to the ADHD per se. (For detailed suggestions on how to monitor and manage sleep disturbances due to adverse medication effects in ADHD children, see [23]).

As a general conclusion, we note that our results are in line with previous findings in children with a diagnosis of ADHD, and indicate that these children present more sleep problems than typically developing children. However, these results may reflect misdiagnoses, and thus special attention should be directed to the differential diagnosis between sleep disturbances and $\mathrm{ADHD} / \mathrm{HKD}$, as well as to the detection of comorbid sleep-related problems. These results have important implications. In line with what others have concluded, based on clinical research findings, we consider that the assessment and improvement of sleep habits should become a routine part of the clinical 
assessment and intervention plans for children referred for $\mathrm{ADHD} / \mathrm{HKD}$, and if necessary, further sleep exams should be undertaken. Either to avoid misdiagnosis or to improve the symptoms of ADHD and sleep-related problems, clinicians should become aware of the necessity for appropriate assessment and treatment of sleep problems in children referred for ADHD.

As well as strengths, the present study has a number of limitations. The sleep questionnaire, although it is a consistent, validated, and carefully-built tool $[32,33]$, does not address neither PLMD, nor RLS. Research suggests that PLMD, RLS and ADHD symptoms may be especially related $[9,13,46]$. In spite of this limitation, children diagnosed with HKD in our sample had more symptoms of sleep-related bruxism, which, like PLMD or RLS, is a disorder currently included in the category of Sleep-Related Movement Disorders by the ICSD-2 [41]. Authorization to conduct the study was restricted in time, which resulted in a limited clinical sample size. We did not control for comorbidities that may contribute in an additive manner to the sleep-related problems of HKD/ADHD (e.g., tic disorders [47]). It was not possible to discern the ICD-10 diagnostic subcategory of HKD ascribed to each child in the clinical sample. We used an HKD sample, which, although it limits comparisons with ADHD samples, constitutes a relevant strength, in that few studies on sleep and hyperactivity have adopted ICD-10 criteria (most have used the DSM criteria). This may be also be viewed as a strength if we consider that we were studying a more homogenous clinical condition, corresponding to a specific ADHD subtype, as defined by the DMS-IV-TR (most probably the combined subtype), instead of a heterogeneous ADHD sample comprising different subtypes. Thus, we believe our study brings insights that are particularly relevant for clinical practice in settings where the ICD-10 is used to diagnose mental disorders. As in other studies (e.g., [38]), one possible technical 
concern from a research point of view was that children were not diagnosed through structured interviews, but instead according to a routine clinical evaluation by a child and adolescent psychiatrist, using anamnesis (and videorecordings in natural environments if necessary). However, such methodology has the potential to guarantee higher ecological validity. We assessed sleep using questionnaires, which are subject to recall bias; ideally, objective methods should be used. However, since subjective sleep complaints are clinically relevant but not necessarily captured by methods such as PSG or actigraphy, sleep questionnaires are essential tools. Our research contained a number of strengths in addition to the ones previously mentioned. In spite of the growing number of published studies of sleep and ADHD/HKD, relatively few have compared medicated and unmedicated children; more importantly, only a limited number have compared clinical samples and matched controls. Therefore, we believe the current study represents an important contribution and calls attention to the higher probability of sleep-related problems in children receiving a diagnosis of HKD. In sum, health professionals assessing children referred due to $\mathrm{ADHD} / \mathrm{HKD}$ should also address sleeprelated issues, in order to detect comorbid sleep conditions or co-occurring sleep complaints that deserve clinical attention and appropriate treatment, or to prevent misdiagnosis.

Acknowledgements: We are deeply grateful to Parents who agreed to participate in this study about their children. Part of this study was developed for the Master Degree dissertation of Dr. Carla Parchão in psychology (U. Aveiro), with the supervision of the first author. Results from this study have been previously presented at the European Sleep Research Congress in 2012, with support from the Research and Development Unit IBILI (FM-UC), FCT Portugal (Portuguese Science and Technology Foundation). 
Conflict of interest statement: On behalf of all authors, the corresponding author states that there is no conflict of interest.

Summary: The aim of this study was to compare sleep, as reported by parents, in children diagnosed with ICD-10 Hyperkinetic Disorder (HKD) versus children in the community, and also to study sleep differences between medicated versus unmedicated HKD children. Parents filled out a previously validated sleep-wake questionnaire. Thirty children, aged 5 to 13 years ( $83.3 \%$ boys), who had been diagnosed with HKD by their child and adolescent psychiatrists, took part in the study. A comparison group, matched for sex, age, and school year, were selected from a large community sample. Statistically significant differences $(\mathrm{p}<.05)$ indicated that HKD children, compared to controls, had later bedtimes, stronger bedtime resistance, longer sleep latency; more often fell asleep in their parents bed and needed something special in order to fall asleep; slept for less time; had more frequent symptoms of nightmares, sleep talking, sleep bruxism, being afraid of the dark, bedwetting and, most notably, loud snoring (26.7\%); displayed higher fatigue and irritability during the day; and tended to show higher somnolence. Comparing HKD children taking methylphenidate $(n=13)$ versus unmedicated children $(\mathrm{n}=17)$, the former had higher bedtime resistance, tended to present later bedtimes and had more nightmares, but appeared to more easily return to sleep autonomously. Our results replicate in HKD children previous findings in ADHD children, and indicate that these children may have more sleep problems than children with typical development. Alternatively, our results may reflect misdiagnoses, thus, these results have important implications. Both to prevent misdiagnosis and to select the best treatment options, special attention should be directed to comorbidity and differential diagnosis issues between sleep disturbances and ADHD/HKD in children. 


\section{References}

[1] American Psychiatric Association (2000) Diagnostic and statistical manual of mental disorders. Text revision (DSM-IV-TR). 4th ed.: APA, Washington, DC

[2] World Health Organization (1992) The tenth revision of the international classification of diseases and related health problems (ICD-10). WHO, Geneva

[3] Cameron M, Hill P (1996) Hyperkinetic disorder: assessment and treatment. Advances in Psychiatric Treatment 2:94-102

[4] Lee SI, Schachar RJ, Chen SX, Ornstein TJ, Charach A, Barr C, Ickowicz A (2008) Predictive validity of DSM-IV and ICD-10 criteria for ADHD and hyperkinetic disorder J Child Psychol Psychiat 49:70-78

[5] Overmeyer S, Taylor E (1999) Annotation: Principles of treatment of hyperkinetic disorder: practice approaches for the U.K. J Child Psychol Psychiat 40:147-175

[6] Döpfner M, Breuer D, Wille N, Erhart M, Ravens-Sieberer U (2008) How often do children meet ICD-10/DSM-IV criteria of attention deficit/hyperactivity disorder and hyperkinetic disorder? Parent-based prevalence rates in a national sample results of the BELLA study. Eur Child Adolesc Psychiatry 17:59-70

[7] Lahey B, Pelham WE, Chronis A, Massetti G, Kipp H, Ehrhardt A, Lee S (2006) Predictive validity of ICD-10 hyperkinetic disorder relative to DSM-IV attentiondeficit/hyperactivity disorder among younger children. J Child Psychol Psyc $47: 472-479$

[8] Hansen BH, Skirbekk B, Oerbeck B, Richter J, Kristensen H (2011) Comparison of sleep problems in children with anxiety and attention deficit/hyperactivity disorders. Eur Child Adolesc Psychiatry 20:321-330

[9] Konafal E, Lecendreux M, Cortese S (2010) Sleep and ADHD. Sleep Med 11:652- 
658

[10] Cortese S, Faraone S, Konofal E, Lecendreux M (2009) Sleep in children with attention-deficit/hyperactivity disorder: meta-analysis of subjective and objective studies J Am Acad Child Adolesc Psychiatry 48:894-908

[11] Walters A, Silvestri R, Zucconi M, Chandrashekariah R, Konofal E (2008) Review of the possible relationship and hypothetical links between attention deficit hyperactivity disorder (ADHD) and the simple sleep related movement disorders, parasomnias, hypersomnias, and circadian rhythm disorders. J Clin Sleep Med 4:591-600

[12] Yoon SY, Jain U, Shapiro C (2012) Sleep in attention-deficit/hyperactivity disorder in children and adults: Past, present, and future. Sleep Med Rev 16:371-388

[13] Sadeh A, Pergamin L, Bar-Haim Y (2006) Sleep in children with attention-deficit hyperactivity disorder: A meta-analysis of polysomnographic studies. Sleep Med Rev 10:381-398

[14] Goodlin-Jones BL, Waters S, Anders TF (2009) Objective sleep measurement in typically and atypically developing preschool children with ADHD-like profiles. Child Psychiat Hum D 40:257-268

[15] Prihodova I, Paclt I, Kemlink D, Skibova J, Ptacek, R, Nevsimalova S (2010) Sleep disorders and daytime sleepiness in children with attention-deficit/hyperactivity disorder: A two-night polysomnographic study with a multiple sleep latency test. Sleep Med 11:922-928

[16] Wagner J, Schlarb AA (2012) Subtypes of ADHD and their association with sleep disturbances in children. Somnologie 16:118-124

[17] Lebourgeois MK, Avis K, Mixon M, et al (2004) Snoring, sleep quality, and sleepiness across attention-deficit/hyperactivity disorder subtypes. Sleep 27:520- 
525

[18] Lecendreux M, Konofal E, Bouvard M, et al (2000) Sleep and alertness in children with ADHD. J Child Psychol Psyc 41:803-812

[19] Mayes SD, Calhoun SL, Bixler EO, et al (2009) ADHD subtypes and comorbid anxiety, depression, and oppositional-defiant disorder: differences in sleep problems. J Pediatr Psychol 34:328-337

[20] Willoughby M, Angold A, Egger H (2008) Parent-reported attentiondeficit/hyperactivity disorder symptomatology and sleep problems in a preschoolage pediatric clinic sample. J Am Acad Child Adolesc Psychiatry 47:1086-1094

[21] Chiang HL, Gau SF-G, Ni H-C, Chiu Y-N, Shang C-Y, Wu Y-Y, et al. (2010) Association between symptoms and subtypes of attention-deficit hyperactivity disorder and sleep problems/disorders. J Sleep Res, 19: 535-545

[22] Cockcroft K, Ashwal J, Bentley A (2009) Sleep and daytime sleepiness in methylphenidate medicated and un-medicated children with attentiondeficit/hyperactivity disorder (ADHD) Afr J Psychiatry 12:275-279

[23] Graham J, Banaschewski T, Buitelaar J, Coghill D, Danckaerts M, Dittmann RW (2011) European guidelines on managing adverse effects of medication for ADHD. Eur Child Adolesc Psychiatry 20:17-37

[24] Cortese S, Holtmann M, Banaschewski T, Buitelaar J, Coghill D, Danckaerts M, et al. and on behalf of the European ADHD Guidelines Group (2013) Practitioner Review: Current best practice in the management of adverse events during treatment with ADHD medications in children and adolescents. J Child Psychol Psychiat 54:227-246

[25] Sangal RB, Owens J, Allen A J, Sutton V, Schuh K, Kelsey D (2006) Effects of atomoxetine and methylphenidate on sleep in children with ADHD. Sleep 29:1573- 


\section{5}

[26] Pressman R, Imber S (2011) Relationship of children's daytime behavior problems with bedtime routines/practices: a family context and the consideration of fauxADHD. Am J Fam Ther 39:404-418

[27] Attention deficit hyperactivity disorder and sleep: Insomnia and other sleep problems may worsen symptoms of ADHD; treatment options exist (2010) Harv Ment Health Lett 27:6-7

[28] Kirov R, Uebel H, Albrecht B, Banaschewski T, Yordanova J, Rothenberger A (2012) Attention-deficit/hyperactivity disorder (ADHD) and adaptation night as determinants of sleep patterns in children. Eur Child Adolesc Psychiatry 21:681690

[29] Cassels T (2013) ADHD, sleep problems, and bed sharing: future considerations. Am J Fam Ther 41:13-25

[30] O'Brien LM, Holbrook CR, Mervis CB, Klaus CJ, Bruner JL, Raffield TJ, . . . Gozal D (2003). Sleep and neurobehavioral characteristics of 5- to 7-year-old children with parentally reported symptoms of attention-deficit/hyperactivity disorder. Pediatrics 111:554-563

[31] Clemente VM (1997) Sono e vigília em crianças de idade escolar: hábitos, comportamentos e problemas. Dissertation. University of Coimbra

[32] Ferreira AM, Clemente V, Gozal D, Gomes A, Pissara C, César H, et al. (2000) Snoring in Portuguese primary school children. Pediatrics 106:64-69

[33] Bos C, Gomes A, Clemente V, Marques M, Pereira AT, Maia B, et al. (2009) Sleep and behavioural/emotional problems in children: A population-based study. Sleep Med 10:66-74

[34] Wechsler D (2003) Escala de Inteligência de Wechsler para Crianças - Terceira 
Edição (WISC-III): Manual [Portuguese manual for the Wechsler Intelligence Scale for Children - third edition]. Cegoc, Lisboa

[35] Wechsler D (2003) Escala de Inteligência de Wechsler para a Idade Pré-Escolar e Primária - Forma Revista (WPPSI-R): Manual [Portuguese manual for the Wechsler Preschool and Primary Scale of Intelligence - revised (WPPSI-R)]. Cegoc, Lisboa

[36] Almeida AC (2011) Sintomas de hiperactividade/défice de atenção e padrões de sono: estudo com crianças dos $1^{\circ}$ e $2^{\circ}$ ciclos [ADHD symptoms and sleep patterns: a survey in primary school children]. Dissertation. Universidade de Aveiro

[37] Hvolby A, Jørgensen J, Bilenberg N (2009) Parental rating of sleep in children with attention deficit/hyperactivity disorder . Eur Child Adolesc Psychiatry 18:429438

[38] Ivanenko A, Crabtree VM, O’Brien LM, Gozal D (2006) Sleep complaints and psychiatric symptoms in children evaluated at a pediatric mental health clinic. J Clin Sleep Med 2:42-48

[39] Weiss MD, Salpekar J (2010) Sleep problems in the child with attention-deficit hyperactivity disorder. Defining aetiology and appropriate treatments. CNS Drugs 24:811-828

[40] Schredl M, Sartorius H (2010) Dream recall and dream content in children with attention deficit/hyperactivity disorder. Child Psychiat Hum D 41:230-238

[41] American Academy of Sleep Medicine (2005) ICSD - International classification of sleep disorders, 2nd ed: Diagnostic and coding manual. AASM, Westchester, Il

[42] Aparas T, Gomes AA, Clemente V \& Azevedo MH (2010) Sono-Vigília em Crianças com Distúrbios do Espectro do Autismo [Sleep-wake patterns in children with autistic spectrum disorders]. Int J Dev Educ Psychol 3:525-533 
[43] Chervin RD, Archbold KH (2001) Hyperactivity and polysomnographic findings in children evaluated for sleep-disordered breathing. Sleep 24:313-320

[44] Chervin RD, Ruzicka DL, Archbold KH, Dillon JE (2005) Snoring predicts hyperactivity four years later. Sleep 28:885-890

[45] Marcus CL, Moore RH, Rosen CL, Giordani B, Garetz, SL, Taylor, HG, et al. (2013). A randomized trial of adenotonsillectomy for childhood sleep apnea. N Engl J Med, 368: 2366-2376

[46] Gaultney JF, Terrell DF, Gingras JL (2005) Parent-reported periodic limb movement, sleep disordered breathing, bedtime resistance behaviors, and ADHD. Behav Sleep Med 3:32-43

[47] Kirov R, Kinkelbur J, Banaschewski T, Rothenberger A (2007) Sleep patterns in children with attention-deficit/hyperactivity disorder, tic disorder, and comorbidity. J Child Psychol Psyc 48:561-570 
Table 1: sleep-wake patterns (medians and means) and difficulties (percentages) in HKD versus matched children

\begin{tabular}{|c|c|c|c|c|c|c|c|}
\hline & HKD & Controls & $\begin{array}{l}\text { Mann- } \\
\text { Withney }\end{array}$ & Cut-off & HKD & $\begin{array}{l}\text { Cont } \\
\text { rols }\end{array}$ & $\begin{array}{l}\text { Fischer } \\
\text { exact test }\end{array}$ \\
\hline sleep-wake schedules and sleep duration & $M d(M)$ & $M d(M)$ & $P$ & & $\%$ & $\%$ & $P$ \\
\hline Bedtime (school nights) & $21: 30(21: 47)$ & $21: 30(21: 12)$ & $<.01$ & - & - & - & - \\
\hline Bedtime (weekend nights) & $22: 30(22: 34)$ & $22: 00(21: 56)$ & $<.05$ & - & - & - & - \\
\hline Rise time (week nights) & 07:45 (07:40) & $7: 30(7: 34)$ & $N S$ & - & - & - & - \\
\hline Rise time (weekend nights) & 09:00 (08:56) & $8: 30(8: 39)$ & $N S$ & - & - & - & - \\
\hline Sleep length (school nights) & 09:00 (09:07) & $10: 00(9: 46)$ & $<.01$ & - & - & - & - \\
\hline Sleep length (weekend nights) & 09:30 (09:38) & $10: 00(10: 15)$ & $<.05$ & - & - & - & - \\
\hline \multicolumn{8}{|l|}{ Initiating and maintaining sleep patterns } \\
\hline Willingness to go to bed & $2.00(2.33)$ & $4.00(3.47)$ & $<.001$ & (a) & 63.3 & 13.3 & $<.001$ \\
\hline Bedtime refusal & $2.00(2.30)$ & $1.00(1.27)$ & $<.001$ & (b) & 40.0 & 3.3 & $<.01$ \\
\hline Fall asleep alone in his/her own bed & $2.00(2.50)$ & $4.00(3.27)$ & $<.05$ & (b) & 53.3 & 23.3 & $<.05$ \\
\hline Falls asleep in parents bed & $2.00(2.40)$ & $1.00(1.63)$ & $<.01$ & (b) & 40.0 & 16.7 & $<.05$ \\
\hline Parents' presence in the room to fall asleep & $2.00(2.21)$ & $1.00(1.70)$ & $<.05$ & (b) & 31.0 & 23.3 & $N S$ \\
\hline Comforting activities/objects to fall asleep & $1.00(2.00)$ & $1.00(1.23)$ & $<.01$ & (b) & 33.3 & 10.0 & $<.05$ \\
\hline Lights on to fall asleep & $1.50(2.23)$ & $1.00(1.77)$ & .125 & (b) & 36.7 & 26.7 & $N S$ \\
\hline Time to fall asleep & $2.00(1.97)$ & $2.00(1.60)$ & $<.05$ & $(>30 \mathrm{~min})$ & 23.3 & 3.3 & $<.05$ \\
\hline Night awakenings & $1.00(0.86)$ & $1.00(1.83)$ & $N S$ & (2 or more) & 16.7 & 10.0 & $N S$ \\
\hline Resumption sleep by her/his own & $4.00(3.00)$ & $4.00(3.43)$ & $N S$ & (a) & 34.6 & 16.7 & .108 \\
\hline \multicolumn{8}{|l|}{ Night behaviors, movements and fears } \\
\hline Loud Snoring & $2.00(2.07)$ & $1.00(1.40)$ & $<.01$ & (b) & 26.7 & 3.3 & $<.05$ \\
\hline Bed wetting & $1.00(1.57)$ & $1.00(1.03)$ & $<.01$ & (b) & 16.7 & 0.0 & $<.05$ \\
\hline Nightmares & $2.00(2.17)$ & $2.00(1.63)$ & $<.05$ & (b) & 33.3 & 3.3 & $<.01$ \\
\hline Sleepwalking & $1.00(1.20)$ & $1.00(1.03)$ & .085 & (b) & 3.3 & 0.0 & $N S$ \\
\hline Sleep talking & $2.00(2.10)$ & $1.00(1.47)$ & $<.01$ & (b) & 33.3 & 0.0 & $<.001$ \\
\hline Night Terrors & $1.00(1.48)$ & $1.00(1.17)$ & .051 & (b) & 10.3 & 0.0 & .112 \\
\hline Teeth grinding & $1.00(1.63)$ & $1.00(1.23)$ & $<.05$ & (b) & 16.7 & 10.0 & $N S$ \\
\hline Fear from darkness & $2.00(2.52)$ & $1.00(1.60)$ & $<.01$ & (b) & 44.8 & 20.0 & $<.05$ \\
\hline \multicolumn{8}{|l|}{ Daytime sleep and complaints } \\
\hline Naps & $1.00(1.23)$ & $1.00(1.13)$ & $N S$ & - & - & - & - \\
\hline Daytime Sleepiness & $1.00(1.53)$ & $1.00(1.27)$ & .103 & - & - & - & - \\
\hline Daytime fatigue & $2.00(2.03)$ & $1.50(1.60)$ & $<.05$ & - & - & - & - \\
\hline Daytime irritability & $2.00(2.17)$ & $2.00(1.67)$ & $<.05$ & - & - & - & - \\
\hline Perceived existence of sleep problems & - & - & - & & & & \\
\hline Child has a sleep problem & - & - & - & (c) & 30 & 6.9 & $<.05$ \\
\hline Seek help for child sleep problem & - & - & - & (c) & 17.2 & 10.0 & $N S$ \\
\hline Take medication to sleep & - & - & - & (c) & 3.3 & 3.3 & $N S$ \\
\hline
\end{tabular}


Table 2: sleep-wake patterns (medians and means) and difficulties (percentages) between non-medicated ( $\mathrm{n}=17)$ and medicated $(\mathrm{n}=13) \mathrm{HKD}$ children [non relevant results were omitted]

\begin{tabular}{|c|c|c|c|c|c|c|c|}
\hline & Unmedicated & Medicated & $\begin{array}{l}\text { Mann- } \\
\text { Withney }\end{array}$ & $\begin{array}{l}\text { Cut- } \\
\text { off } 1\end{array}$ & $\begin{array}{c}\text { Un } \\
\text { edicated }\end{array}$ & $\begin{array}{l}\text { Medica- } \\
\text { ted }\end{array}$ & $\begin{array}{l}\text { Fischer } \\
\text { exact test }\end{array}$ \\
\hline Sleep-wake schedules and sleep duration & $M d(M)$ & $M d(M)$ & $P$ & & $\%$ & $\%$ & $P$ \\
\hline Bedtime (school nights) & $21: 30(21: 33) 2$ & $22: 00(22: 05)$ & .052 & - & - & - & - \\
\hline Bedtime (weekend nights) & $22: 15(22: 18) 2$ & $23: 00(22: 55)$ & 0.05 & - & - & - & - \\
\hline \multicolumn{8}{|l|}{ Initiating and maintaining sleep patterns } \\
\hline Willingness to go to bed & $2.00(2.71)$ & $2.00(1.85)$ & $<.05$ & (a) & 52.9 & 76.9 & .167 \\
\hline Bedtime refusal & $2.00(1.94)$ & $3.00(2.77)$ & .053 & (b) & 29.4 & 53.8 & .164 \\
\hline Lights on to fall asleep & $1.00(1.88)$ & $4.00(2.69)$ & .153 & (b) & 23.5 & 53.8 & .093 \\
\hline Resumption sleep by her/his own & $2.50(2.57)$ & $4.00(3.50)$ & .065 & (a) & 50.0 & 16.7 & .085 \\
\hline \multicolumn{8}{|l|}{ Night behaviors, movements and fears } \\
\hline Nightmares & $2.00(1.94)$ & $3.00(2.46)$ & .083 & (b) & 17.6 & 53.8 & $<.05$ \\
\hline Fear from darkness & $2.00(2.25)$ & $3.00(2.85)$ & .188 & - & - & - & - \\
\hline \multicolumn{8}{|l|}{ Daytime sleep and complaints } \\
\hline Daytime fatigue & $2.00(1.82)$ & $2.00(2.31)$ & .103 & - & - & - & - \\
\hline \multicolumn{8}{|l|}{ Perceived existence of sleep problems } \\
\hline Child has a sleep problem & - & - & & (c) & 11.8 & 53.8 & $<0.05$ \\
\hline Seek help for child sleep problem & - & - & & (c) & 6.2 & 30.8 & .144 \\
\hline
\end{tabular}

